\title{
Comparison between serum magnesium levels of asphyxiated neonates and normal cases
}

\author{
Nasrin Khalessi ${ }^{1}$, Ali Mazouri ${ }^{2}$, Mandana Bassirnia ${ }^{1}$, Ladan Afsharkhas ${ }^{1 *}$ \\ Received: 03 Jan 2016 \\ Published: 9 Mar 2017
}

\begin{abstract}
Background: Asphyxia is one the most important causes of neonatal mortality and morbidity. It is suggested that magnesium may have a protective role against cellular damage during hypoxic brain insult, or change effect post-asphyxia consequences. Our study was performed for comparison of serum magnesium in neonates with and without asphyxia.

Methods: This study was done in Neonatal Ward of Ali-Asghar hospital, from January 2010 to 2011 in Tehran, Iran. Serum magnesium levels of seventy-six cases with a diagnosis of asphyxia grade 2 were compared with 76 normal newborns. Collected data including gestational age, sex, birth weight and serum magnesium levels were analyzed by SPSS software.

Results: Of 152 neonates, 81 (53.3\%) were male. Mean gestational age was $37.9 \pm 1.07$ weeks. Mean birth weights were $3172.9 \pm$ 411.20 grams. Mean serum magnesium levels were compared in asphyxiated and normal neonates and between two groups significant difference was found $(\mathrm{p}=0.01)$. The odds ratio was 2.188 (with lower1.826, upper 2.626 and confidence interval 95 percent) which suggested a significant correlation between asphyxia and hypomagnesemia.

Conclusion: This study showed that serum magnesium levels in neonates with asphyxia was significantly lower than normal neonates and asphyxia can lead to hypomagnesemia.
\end{abstract}

Keywords: Asphyxia, Magnesium, Neonate

Copyright $@$ Iran University of Medical Sciences

Cite this article as: Khalessi N, Mazouri A, Bassirnia M, Afsharkhas L. Comparison between serum magnesium levels of asphyxiated neonates and normal cases. Med J Islam Repub Iran. 2017 (9 Mar);31:19. https://doi.org/10.18869/mjiri.31.19

\section{Introduction}

Asphyxia is a medical condition in which embryonic hypoxia and hypercapnia occur due to the deprivation of oxygen to a newborn. This condition occurs during the first and the second phase of delivery which is recognized by embryonic acidosis (umbilical cord PH less than 7). Prenatal asphyxia is one of the most common causes of neonatal mortality. According to the available data, nearly $20 \%$ of neonatal mortality is due to prenatal asphyxia (1). Asphyxia is characterized by signs of neonatal encephalopathy (hypotonia, declined neonatal reflexes, status of pupils and seizure), Apgar score $<4$ at first and Apgar score $<7$ at fifth minutes of birth and umbilical cord $\mathrm{PH}$ less than 7 (2).

Prenatal asphyxia results in hypoxic damage in different body organs including kidneys, lungs and liver, but its most serious impact concerns the central nervous system (CNS). In the cellular level, hypoxic -ischemic damage

Corresponding author:Dr Ladan Afsharkhas,dr.afsharkhas1@yahoo.com

1. Ali-Asghar Children's Hospital, Iran University of Medical Science, Tehran, Iran.

2. Akbarabadi Maternity Hospital, Iran University of Medical Science, Tehran, Iran. results in a flood of a large number of biochemical changes which lead to cell death (3).

Magnesium is mainly an intra-cellular action, and very low amount of its total volume exists in extra-cellular space. Magnesium concentration remains stable within the narrow space of cell membrane by strong mechanisms of absorption and excretion of magnesium. In normal condition, magnesium exchange between intra- and extra- cellular space occurs slowly, whereas, in the ischemic condition, the speed of this exchange rises (4).

Neonatal asphyxia leads to the production of high levels of glutamate amino acid in the brain. Within the first 72 hours, a large amount of glutamate causes irreversible neuronal damage by activating certain receptors and influx of calcium into neurons. Magnesium blocks these receptors and has a neuroprotective role which blocks the impact of asphyxia on different body organs (5).

$\uparrow$ What is "already known" in this topic:

Asphyxia is a neonatal encephalopathy with high rate of morbidity and mortality. Magnesium has an important and protective role in central nervous system (CNS).

$\rightarrow$ What this article adds:

In asphyxiated patients, serum magnesium decreases and detection - and in next studies treatment- of it may help to prevention of CNS complications. 
Asphyxia may act as a risk factor of Intracranial hemorrhages $(\mathrm{ICH})$ in term newborns although $\mathrm{ICH}$ is more common in preterm infants $(6,7)$. Magnesium supplements have protective effects against neurologic complications of ICH in acutely ill neonates $(8,9)$.

This study is designed to evaluate serum magnesium levels in neonates with asphyxia, and there was no study about this subject in Iran. Detection of hypomagnesemia in asphyxiated neonates will be the base of our future interventional studies in these high-risk infants.

\section{Methods}

Our research was a case-control study and performed in neonatal ward of Ali-Asghar children Hospital -tertiary and training center in Tehran, Iran-from January 2010 to January 2011.

The study was started after Ethical Committee of Iran University approved it (research code: 2248). The consent forms were filled by parents. During the study period, 152 neonates including 76 cases with a diagnosis of asphyxia and 76 normal newborns were compared.

Asphyxiated neonates were visited by a neonatologist in Akbarabadi Maternity Hospital and referred to our center. In Ali-Asghar NICU, patients were visited by second neonatologist and severity of asphyxia re-evaluated according to Sarnat and Sarnat classification. Perinatal asphyxia from the point of severity has three stages: In stage 1 there are mild encephalopathy, infant hyperalertness, dilated pupils and normal electroencephalography (EEG). Stage 2 includes moderate encephalopathy, hypotonia, lethargy, small pupils, seizures and abnormal EEG. In stage 3, there are severe encephalopathy, flaccidity, absence of reflexes, stupor, seizures and abnormal EEG with decreased background activity $(1,2)$. Only cases with stage 2 asphyxia were enrolled in the study. Control cases were selected randomly from normal neonates with age of 3-5 days that examined in Ali-Asghar neonatal clinic as a routine care and matched for age and sex.

Exclusion criteria were defined as total parenteral nutrition (TPN), pre-eclampsia and magnesium sulfate therapy, prematurity, infants of diabetic mothers, neonatal systemic infections and metabolic disorders. During 24 hours after birth, $2 \mathrm{ml}$ venous blood samples were taken. Blood specimens were collected and serum magnesium levels measured. Values of $1.5-2.3 \mathrm{mg} / \mathrm{dl}$ as normal ranges of serum magnesium was considered. Data about gestational age, sex, and birth weight were recorded. The collected data was analyzed using $16^{\text {th }}$ version of statistical package for social sience (SPSS). Descriptive Statistical including frequency, mean and standard deviation and analytical statistics including Chi-square (qualitative variables comparison), t-test (quantitative variables comparison) and Odd ratios (at a confidence level of $95 \%$ ) was calculated.
$P$ values less than 0.05 was considered significant.

\section{Results}

Among 152 neonates, 81(53.3\%) were male. Mean gestational age was $37.9 \pm 1.07$ week (range 37 to 40 week). Mean birth weights were $3172.9 \pm 411.20$ grams. Mean serum magnesium level was $1.89 \pm 0.32 \mathrm{mg} / \mathrm{dl}$.). Serum levels of magnesium in boys and girls didn't have significant difference $(1.96 \pm 0.33 \mathrm{mg} / \mathrm{dl}$ vs. $1.92 \pm 0.30 \mathrm{mg} / \mathrm{dl}$, $\mathrm{p}=0.8$ ). Besides, no significant difference was seen between serum magnesium levels and gestational age and weight $(\mathrm{p}=0.8$ and $\mathrm{p}=0.3)$. Mean serum magnesium level, gestational age, sex, and birth weight were compared in asphyxiated and normal neonates. The results was showed in Table 1. There was a significant difference between serum magnesium levels of two mentioned groups $(p=0.01)$. Twelve $(16 \%)$ of neonates with asphyxia had hypomagnesemia. There was no case of hypomagnesemia in normal group $(\mathrm{p}=0.001)$.

The odds ratio was 2.188 with lower 1.826 , upper 2.626 and confidence interval 95 percent which suggested a significant correlation between asphyxia and hypomagnesemia.

\section{Discussion}

Studies have shown some metabolic abnormalities in asphyxiated neonates including hypoglycemia, hyperammonemia, hypocalcemia and hypomagnesemia. Some of these disturbances lead to seizures and more brain injury $(10,11)$.

Geven and Tsang's studies showed that asphyxia at birth leads to hypomagnesemia $(5,12)$. Jukarinen reported hypermagnesemia followed by asphyxia in their studies (13). The underlying cause of these changes in total magnesium levels in asphyxiated neonates is not clear yet. Although in our study mean magnesium levels in asphyxiated cases was around $1.9 \mathrm{mg} / \mathrm{dl}$, being in its normal range which cannot be interpreted as hypo or hypermagnesemia, hypomagnesemia was detected only in asphyxiated cases.

In a study conducted by Ilves et al, total magnesium concentration and ionized calcium in neonates with hypoxic-ischemic encephalopathy was evaluated. It was shown that asphyxiated neonates with or without signs of mild hypoxic-ischemic encephalopathy have higher total magnesium levels $(0.81 \mathrm{mmol} / \mathrm{l}=1.96 \mathrm{mg} / \mathrm{dl})$, while neonates with severe hypoxic-ischemic encephalopathy have lower total magnesium level $(0.64 \mathrm{mmol} / \mathrm{l}=1.55 \mathrm{mg} / \mathrm{dl})$ in comparison with the control group $(0.72 \mathrm{mmol} / \mathrm{l}=1.75$ $\mathrm{mg} / \mathrm{dl})(\mathrm{p}<0.05)(3)$.

In a study designed by Bhat et al, 40 term asphyxiated neonates were examined and mean serum magnesium levels in asphyxiated cases was near to the result of our study. This similarity indicates that this number can be

Table 1. Comparison of characteristics and serum magnesium in two groups

\begin{tabular}{|c|c|c|c|}
\hline Variable & Asphyxiate neonates $(n=76)$ & Normal neonates $(n=76)$ & P value \\
\hline Birth weight (gr) & $3130.1 \pm 421.07^{*}$ & $3215.8 \pm 399.27^{*}$ & 0.20 \\
\hline Gestational age (w) & $37.96 \pm 1.14 *$ & $37.8 \pm 1.13 *$ & 0.49 \\
\hline Serum magnesium $(\mathrm{mg} / \mathrm{dl})$ & $1.87 \pm 0.37 *$ & $2.0 \pm 0.24 *$ & 0.01 \\
\hline
\end{tabular}

*Mean \pm SD 
considered as basic magnesium levels in neonates with asphyxia; however, further studies should be carried out to prove this issue (4).

In the present study, serum magnesium levels in asphyxiated neonates were significantly lower than normal neonates and hypomagnesemia detected only in asphyxiated patients. In McIntosh et al. study, demonstrated that rapid decline of brain magnesium ions concentrations in rats occurs during hypoxic brain damage and can be correlated with severity of the injury. In their study, ATP$\mathrm{MgCl}_{2}$ had a beneficial effect on neurological function (14).

Hoffman et al. in an experimental study showed that activity of Na-K ATPase, as a marker of neuron membrane function, in asphyxiated cases decreases and leads to neuronal dysfunction. They tried magnesium sulfate after hypoxic injury and compared it with a control group. Upper levels of plasma magnesium increased mentioned marker and attenuated neuronal dysfunction (15).

In Hossain et al. study, 50 neonates with moderate to severe hypoxic-Ischemic encephalopathy were divided into two groups. Mean serum magnesium levels was $1.6 \pm 0.3$ and $1.8 \pm 0.4 \mathrm{mg} / \mathrm{dl}$ in study and control groups. They received magnesium sulfate and normal saline respectively. Neurological abnormalities were detected in $26 \%$ of experimental cases and $61 \%$ of control group. They demonstrated that magnesium infusion improves short outcome in neonatal asphyxia (16).

In our study, no severe case of asphyxia was seen which might be due to our limitations such as small sample size, or since this hospital is a referral center with no nearby maternity center, less severe cases (with transferring possibility) were admitted.

\section{Conclusion}

Asphyxia can lead to hypomagnesemia, and it is recommended to evaluate levels of magnesium in neonates with asphyxia as a routine test and treat properly any case with decreased serum level of magnesium.

\section{Acknowledgements}

We would like to thank personnel of NICU and nursery of Ali-Asghar and Akbarabadi hospitals for their cooperation.

Conflict of Interests: The authors declare that they have no competing interests.

\section{References}

1. Leuthner SR, Das UG. Low Apgar scores and the definition of birth asphyxia. Pediatr Clin N Am 2004;51(3):737-745.

2. Sarnat HB, Sarnat MS. Neonatal encephalopathy following fetal distress. A clinical and electroencephalographic study. Arch Neurol. 1976;33(10):696-705.

3. Ilves P, Kiisk M, Soopold T, Talvik T. Serum total magnesium and ionized calcium concentrations in asphyxiated term newborn infants with hypoxic-ischaemic encephalopathy. Acta Paediatr. 2000;89(6): 680-5.

4. Bhat MA, Magnesium sulfate in severe perinatal asphyxia: a randomized, placebo-controlled trial. Pediatrics. 2009;23(5):764-9.

5. Geven WB, Monnens LAH, Willems JL. Magnesium metabolism.in childhood. Miner Electrolyte Metab. 1993;19:308-13.

6.Khalessi N, Farahani Z, Shariat M, Rezaeizadeh G. Risk Factors of Intracranial Hemorrhage in Premature Neonates. Acta Med Iran. 2014;52(9):687-689.

7. Afsharkhas L, Khalessi N, Karimi Panah M. Intraventricular Hemorrhage in Term Neonates: Sources, Severity and Outcome. Iran J Child Neurol. 2015;9(3):34-39.

8. Westermaier T, Stetter Ch, Kunze E, Willner N, Raslan F, Vince GH, et al. Magnesium treatment for neuroprotection in ischemic diseases of the brain. Exp Trans Strok Med. 2013;5(6):1-10.

9. Petrova A, Mehta R. Magnesium sulfate tocolysis and intraventricular hemorrhage in very preterm infants. Indian J Pediatr. 2012;79(1):43-7.

10. Saha D, Ali MA, Haque MA, Ahmed MS, Sutradhar PK, Latif T, et al. Association of hypoglycemia, hypocalcemia and hypomagnesemiain neonates with perinatal asphyxia. Mymensingh Med J. 2015;24(2):244-50.

11. Khalessi N, Khosravi N, Mirjafari M, Afsharkhas L. Plasma Ammonia Levels in Newborns with Asphyxia. Iran J Child Neurol Winter. 2016;10(1):42-46.

12. Tsang RC, Steichen JJ, Chan GM. Neonatal hypocalcemia,Mechanism of occurrence and management. Crit Care Med. 1977;5:56-61.

13. Jukarinen J. Plasma magnesium concentrations during the first five days of life. Acta Paediatr Scand. 1971;S222:5-55.

14. McIntosh TK, Vink R, Yamakami I, Faden AI. Magnesium protects against neurological deficit after brain injury. Brain Res. 1989;482(2): 252-260.

15. Hoffman DJ, Marro PJ, McGowan JE, Mishra OP, Papadopoulos MD. Protective effect of MgSO4 infusion on NMDA receptor binding characteristics during cerebral cortical hypoxia in newborn piglet. Brain Res. 1994;644(1):144-149.

16. Hossain MM, Mannan MA, Yeasmin F, Shaha CK, Rahman MH, Shahidullah M. Short-term outcome of magnesium sulfate infusion in perinatal asphyxia. Mymensingh Med J. 2013;22(4):727-35. 